\title{
Macroscopic Description for Networks of Spiking Neurons
}

\author{
Ernest Montbrió, ${ }^{1}$ Diego Pazó, ${ }^{2}$ and Alex Roxin ${ }^{3}$ \\ ${ }^{1}$ Center for Brain and Cognition, Department of Information and Communication Technologies, \\ Universitat Pompeu Fabra, 08018 Barcelona, Spain \\ ${ }^{2}$ Instituto de Física de Cantabria (IFCA), CSIC-Universidad de Cantabria, 39005 Santander, Spain \\ ${ }^{3}$ Centre de Recerca Matemàtica, Campus de Bellaterra, Edifici C, 08193 Bellaterra, Spain
}

(Received 30 December 2014; published 19 June 2015)

\begin{abstract}
A major goal of neuroscience, statistical physics, and nonlinear dynamics is to understand how brain function arises from the collective dynamics of networks of spiking neurons. This challenge has been chiefly addressed through large-scale numerical simulations. Alternatively, researchers have formulated mean-field theories to gain insight into macroscopic states of large neuronal networks in terms of the collective firing activity of the neurons, or the firing rate. However, these theories have not succeeded in establishing an exact correspondence between the firing rate of the network and the underlying microscopic state of the spiking neurons. This has largely constrained the range of applicability of such macroscopic descriptions, particularly when trying to describe neuronal synchronization. Here, we provide the derivation of a set of exact macroscopic equations for a network of spiking neurons. Our results reveal that the spike generation mechanism of individual neurons introduces an effective coupling between two biophysically relevant macroscopic quantities, the firing rate and the mean membrane potential, which together govern the evolution of the neuronal network. The resulting equations exactly describe all possible macroscopic dynamical states of the network, including states of synchronous spiking activity. Finally, we show that the firing-rate description is related, via a conformal map, to a low-dimensional description in terms of the Kuramoto order parameter, called Ott-Antonsen theory. We anticipate that our results will be an important tool in investigating how large networks of spiking neurons self-organize in time to process and encode information in the brain.
\end{abstract}

DOI: 10.1103/PhysRevX.5.021028

Subject Areas: Biological Physics, Interdisciplinary Physics, Nonlinear Dynamics

\section{INTRODUCTION}

The processing and coding of information in the brain necessarily imply the coordinated activity of large ensembles of neurons. Within sensory regions of the cortex, many cells show similar responses to a given stimulus, indicating a high degree of neuronal redundancy at the local level. This suggests that information is encoded in the population response and, hence, can be captured via macroscopic measures of the network activity [1]. Moreover, the collective behavior of large neuronal networks is particularly relevant given that current brain measurement techniques, such as electroencephalography or functional magnetic resonance imaging, provide data that are necessarily averaged over the activity of a large number of neurons.

The macroscopic dynamics of neuronal ensembles has been extensively studied through computational models of

Published by the American Physical Society under the terms of the Creative Commons Attribution 3.0 License. Further distribution of this work must maintain attribution to the author(s) and the published article's title, journal citation, and DOI. large networks of recurrently coupled spiking neurons, including Hodgkin-Huxley-type conductance-based neurons [2], as well as simplified neuron models; see, e.g., Refs. [3-5]. In parallel, researchers have sought to develop statistical descriptions of neuronal networks, mainly in terms of a macroscopic observable that measures the mean rate at which neurons emit spikes, the firing rate [6-20]. These descriptions, called firing-rate equations (FREs), have been proven to be extremely useful in understanding general computational principles underlying functions such as memory [21,22], visual processing [23-25], motor control [26], or decision making [27].

Despite these efforts, to date there is no exact theory linking the dynamics of a large network of spiking neurons with that of the firing rate. Specifically, current macroscopic descriptions do not offer a precise correspondence between the microscopic dynamics of the individual neurons, e.g., individual membrane potentials, and the firing-rate dynamics of the neuronal network.

Indeed, FREs are generally derived through heuristic arguments that rely on the underlying spiking activity of the neurons being asynchronous and hence uncorrelated. As such, firing-rate descriptions are not sufficient to describe 
network states involving some degree of spike synchronization. Synchronization is, however, a ubiquitous phenomenon in the brain, and its potential role in neuronal computation is the subject of intense research [14,28-34]. Hence, the lack of firing-rate descriptions for synchronous states limits the range of applicability of mean-field theories to investigate neuronal dynamics.

Here, we propose a method to derive the FREs for networks of heterogeneous, all-to-all coupled quadratic integrate-and-fire (QIF) neurons, which is exact in the thermodynamic limit, i.e., for large numbers of neurons. We consider an ansatz for the distribution of the neurons' membrane potentials that we denominate the Lorentzian ansatz (LA). The LA solves the corresponding continuity equation exactly, making the system amenable to theoretical analysis. Specifically, for particular distributions of the heterogeneity, the LA yields a system of two ordinary differential equations for the firing rate and mean membrane potential of the neuronal population. These equations fully describe the macroscopic states of the networkincluding synchronized states, and represent the first example of an exact firing-rate description for a network of recurrently connected spiking neurons. Finally, we show how the LA transforms, via a conformal mapping, into the so-called Ott-Antonsen ansatz (OA) that is used extensively to investigate the low-dimensional dynamics of large populations of phase oscillators in terms of the Kuramoto order parameter [35].

\section{MODEL DESCRIPTION}

Hodgkin-Huxley-type neuronal models can be broadly classified into two classes, according to the nature of their transition to spiking in response to an injected current [36,37]. Neuronal models with so-called class I excitability generate action potentials with arbitrarily low frequency, depending on the strength of the applied current. This occurs when a resting state disappears through a saddlenode bifurcation. In contrast, in neurons with class II excitability, the action potentials are generated with a finite frequency. This occurs when the resting state loses stability via a Hopf bifurcation. The QIF neuron is the canonical model for class I neurons, and, thus, generically describes their dynamics near the spiking threshold $[5,38,39]$. Our aim here is to derive the FREs corresponding to a heterogeneous all-to-all coupled population of $N$ QIF neurons. The correspondence is exact in the thermodynamic limit, i.e., when $N \rightarrow \infty$ (this convergence was studied recently in Ref. [40]).

The microscopic state of the population of QIF neurons is given by the membrane potentials $\left\{V_{j}\right\}_{j=1, \ldots, N}$, which obey the following ordinary differential equations [5]:

$$
\dot{V}_{j}=V_{j}^{2}+I_{j}, \quad \text { if } V_{j} \geq V_{p}, \quad \text { then } V_{j} \leftarrow V_{r} .
$$

Here, the overdot denotes the time derivative and $I_{j}$ represents an input current. Each time a neuron's membrane potential $V_{j}$ reaches the peak value $V_{p}$, the neuron emits a spike and its voltage is reset to the value $V_{r}$. In our analysis, we consider the limit $V_{p}=-V_{r} \rightarrow \infty$. This resetting rule captures the spike reset as well as the refractoriness of the neuron. Without loss of generality, we rescale the time and the voltage in Eq. (1) to absorb any coefficients that would have appeared in the first two terms. The form for the input currents is

$$
I_{j}=\eta_{j}+J s(t)+I(t),
$$

where the external input has a heterogeneous, quenched component $\eta_{j}$ as well as a common time-varying component $I(t)$, and the recurrent input is the synaptic weight $J$ times the mean synaptic activation $s(t)$, which is written as

$$
s(t)=\frac{1}{N} \sum_{j=1}^{N} \sum_{k \backslash t_{j}^{k}<t} \int_{-\infty}^{t} d t^{\prime} a_{\tau}\left(t-t^{\prime}\right) \delta\left(t^{\prime}-t_{j}^{k}\right) .
$$

Here, $t_{j}^{k}$ is the time of the $k$ th spike of the $j$ th neuron, $\delta(t)$ is the Dirac delta function, and $a_{\tau}(t)$ is the normalized synaptic activation caused by a single presynaptic spike with time scale $\tau$, e.g., $a_{\tau}(t)=e^{-t / \tau} / \tau$.

\section{A. Continuous formulation}

In the thermodynamic limit $N \rightarrow \infty$, we drop the indices in Eqs. (1) and (2) and denote $\rho(V \mid \eta, t) d V$ as the fraction of neurons with membrane potentials between $V$ and $V+d V$ and parameter $\eta$, at time $t$. Accordingly, parameter $\eta$ now becomes a continuous random variable distributed according to a probability distribution function $g(\eta)$. The total voltage density at time $t$ is then given by $\int_{-\infty}^{\infty} \rho(V \mid \eta, t) g(\eta) d \eta$.

The conservation of the number of neurons leads to the following continuity equation:

$$
\partial_{t} \rho+\partial_{V}\left[\left(V^{2}+\eta+J s+I\right) \rho\right]=0,
$$

where we explicitly include the velocity given by Eqs. (1) and (2).

\section{RESULTS}

The continuity equation (4) without temporal forcing $I(t)=0$ has a trivial stationary solution. For each value of $\eta$, this solution has the form of a Lorentzian function: $\rho_{0}(V \mid \eta) \propto\left(V^{2}+\eta+J_{s}\right)^{-1}$. Physically, the Lorentzian density means that firing neurons with the same $\eta$ value will be scattered with a density inversely proportional to their speed [Eq. (1)]; i.e., they will accumulate at slow places and thin out at fast places on the $V$ axis. In addition, for those $\eta$ values corresponding to quiescent neurons, the 
density $\rho_{0}$ collapses at the rest state in the form of a Dirac delta function.

Next, we assume that, independently of the initial condition, solutions of Eq. (4) generically converge to a Lorentzian-shaped function, so that all relevant dynamics occur inside that lower-dimensional space. This fact is mathematically expressed by the following LA for the conditional density functions:

$$
\rho(V \mid \eta, t)=\frac{1}{\pi} \frac{x(\eta, t)}{[V-y(\eta, t)]^{2}+x(\eta, t)^{2}},
$$

which is a Lorentzian function with time-dependent halfwidth $x(\eta, t)$ and center at $y(\eta, t)$. In the following, we assume that the LA Eq. (5) completely describes the macroscopic dynamics of the network of QIF neurons and postpone the mathematical justification of its validity to Sec. III E.

\section{A. Macroscopic observables: Firing rate and mean membrane potential}

The half-width $x(\eta, t)$ of the LA has a particularly simple relation with the firing rate of the neuronal population (i.e., the number of spikes per unit time). Indeed, the firing rate for each $\eta$ value at time $t, r(\eta, t)$, can be computed by noting that neurons fire at a rate given by the probability flux at infinity: $r(\eta, t)=\rho(V \rightarrow \infty \mid \eta, t) \dot{V}(V \rightarrow \infty \mid \eta, t)$. The limit $V \rightarrow \infty$ on the right-hand side of this equation can be evaluated within the LA, and gives the simple identity

$$
x(\eta, t)=\pi r(\eta, t) .
$$

The (total) firing rate $r(t)$ is then

$$
r(t)=\frac{1}{\pi} \int_{-\infty}^{\infty} x(\eta, t) g(\eta) d \eta
$$

Additionally, the quantity $y(\eta, t)$ is, for each value of $\eta$, the mean of the membrane potential:

$$
y(\eta, t)=\text { p.v. } \int_{-\infty}^{\infty} \rho(V \mid \eta, t) V d V .
$$

Here, we take the Cauchy principal value, defined as p.v. $\int_{-\infty}^{\infty} f(x) d x=\lim _{R \rightarrow \infty} \int_{-R}^{R} f(x) d x$, to avoid the otherwise ill-defined integral. The mean membrane potential is then

$$
v(t)=\int_{-\infty}^{\infty} y(\eta, t) g(\eta) d \eta
$$

\section{B. Firing-rate equations}

Substituting the LA Eq. (5) into the continuity equation (4), we find that, for each value of $\eta$, variables $x$ and $y$ must obey two coupled equations, which can be written in complex form as

$$
\partial_{t} w(\eta, t)=i\left[\eta+J s(t)-w(\eta, t)^{2}+I(t)\right],
$$

where $w(\eta, t) \equiv x(\eta, t)+i y(\eta, t)$. Closing this equation requires expressing the mean synaptic activation $s(t)$ as a function of $w(\eta, t)$. The simplest choice is to take the limit of infinitely fast synapses, $\tau \rightarrow 0$ in Eq. (3), so that we get an equality with the firing rate $s(t)=r(t)$. This allows for the system of QIF neurons [Eqs. (1)-(3)] to be exactly described by Eqs. (10) and (7), an infinite set of integrodifferential equations if $g(\eta)$ is a continuous distribution.

Equation (10) is useful for general distributions $g(\eta)$ (see Appendix B), but a particularly sharp reduction in dimensionality is achieved if $\eta$ is distributed according to a Lorentzian distribution of half-width $\Delta$ centered at $\bar{\eta}$ :

$$
g(\eta)=\frac{1}{\pi} \frac{\Delta}{(\eta-\bar{\eta})^{2}+\Delta^{2}} .
$$

Note that this distribution accounts for the quenched variability in the external inputs. The fact that it is Lorentzian is unrelated to the LA for the density of membrane potentials. Assuming Eq. (11), the integrals in Eqs. (7) and (9) can be evaluated closing the integral contour in the complex $\eta$ plane and using the residue theorem [41]. Notably, the firing rate and the mean membrane potential only depend on the value of $w$ at the pole of $g(\eta)$ in the lower half $\eta$ plane:

$$
\pi r(t)+i v(t)=w(\bar{\eta}-i \Delta, t) .
$$

As a result, we only need to evaluate Eq. (10) at $\eta=\bar{\eta}-i \Delta$, and thereby obtain a system of FREs composed of two ordinary differential equations:

$$
\begin{aligned}
& \dot{r}=\Delta / \pi+2 r v, \\
& \dot{v}=v^{2}+\bar{\eta}+J r+I(t)-\pi^{2} r^{2} .
\end{aligned}
$$

This nonlinear system describes the macroscopic dynamics of the population of QIF neurons in terms of the population firing rate $r$ and mean membrane potential $v$.

It is enlightening to compare the mean-field model Eq. (12) with the equations of the spiking neurons. Note that Eq. (12b) resembles Eqs. (1) and (2) for the individual QIF neuron, but without spike resetting. Indeed, the macroscopic firing-rate variable $r$ enters as a negative feedback term in Eq. (12b) and impedes the explosive growth of the mean membrane potential $v$.

This negative feedback, combined with the coupling term on the right-hand side of Eq. (12a), describes the effective interaction between the firing rate and mean membrane potential at the network level. Therefore, the 
FREs (12) describe the effect of the single-cell spike generation and reset mechanism at the network level.

In the following, we examine the dynamics described by Eq. (12) and show that they fully reproduce the macroscopic dynamics of the network of QIF neurons, even during episodes of strong spike synchrony.

\section{Analysis of the firing-rate equations}

To begin with the analysis of Eq. (12), we first note that in the absence of forcing, $I(t)=0$, the only attractors of Eq. (12) are fixed points. Figure 1(a) shows a phase diagram of the system as a function of the mean external drive $\bar{\eta}$ and synaptic weight $J$, both normalized by the width of the input distribution [42]. There are three qualitatively distinct regions of the phase diagram: (1) a single stable node corresponding to a low-activity state (white), (2) a single stable focus (spiral) generally corresponding to a high-activity state (gray), and (3) a region of bistability between low and high firing rate [cyan; see a phase portrait of this region in Fig. 1(d)]. Comparison of a sample bifurcation diagram of the fixed points from numerical simulation of networks of QIF neurons with that obtained from the FREs (12) shows an excellent correspondence; see Figs. 1(b) and 1(c).

A similar phase diagram can be readily reproduced by traditional heuristic firing-rate models, with one significant qualitative difference: the presence of a stable focus-and hence, damped oscillations. Specifically, in the gray region of the phase diagram in Fig. 1(a), the system undergoes oscillatory decay to the stable fixed point. This oscillatory decay occurs as well for the high-activity state over a large extent of the region of bistability (cyan); see, e.g., Fig. 1(d).

The presence of damped oscillations at the macroscopic level reflects the transitory synchronous firing of a fraction of the neurons in the ensemble. This behavior is common in spiking neuron models with weak noise, and is not captured by traditional firing-rate models (see, e.g., Ref. [20]).

\section{Analysis of the firing-rate equations: Nonstationary inputs}

To show that the FREs (12) fully describe the macroscopic response of the population of QIF neurons to timevarying stimuli (up to finite-size effects), we consider two types of stimulus $I(t)$ : (1) a step function and (2) a sinusoidal forcing. In both cases, we simulate the full system of QIF neurons and the FREs (12).

Figure 2 shows the system's response to the two different inputs. In both cases, the system is initially $(t<0)$ in a bistable regime and set in the low-activity state, with parameters corresponding to those of Fig. 1(d). The lefthand panels of Fig. 2 show the response of the system to a step current applied at $t=0$. The applied current is such that the system abandons the bistable region-see Fig. 1(a)—and approaches the high-activity state, which is a stable focus. This is clearly reflected in the time series $r(t), v(t)$, where the rate equations (12) exactly predict the damped oscillations exhibited by the mean field of the QIF neurons. The raster plot in Fig. 2(e) shows the presence of the oscillations, which is due to the transitory synchronous firing of a large fraction of neurons in the population. Finally, at $t=30$, the current is removed and the system converges-again, showing damped oscillations-to the new location of the (focus) fixed point, which clearly coexists with the stable node where it was originally placed $(t<0)$.

The right-hand panels of Fig. 2 show the response of the model to a periodic current, which drives the system from one side of the bistable region to the other. As a result, we (a)

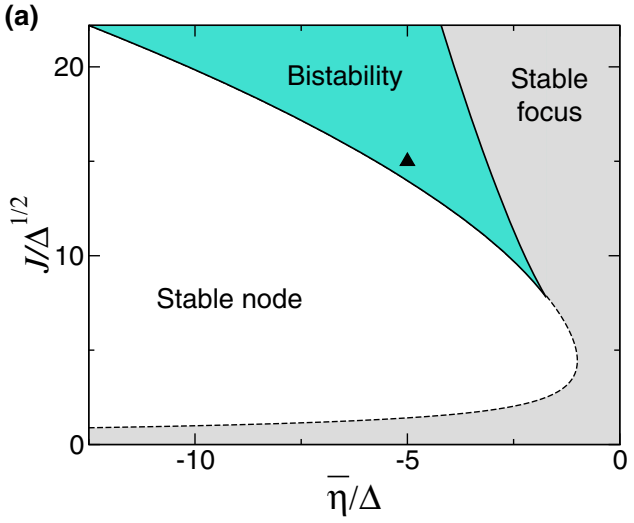

(b)

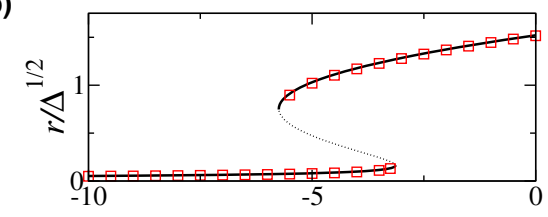

(c)

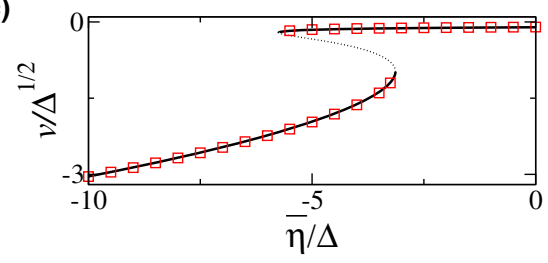

(d)

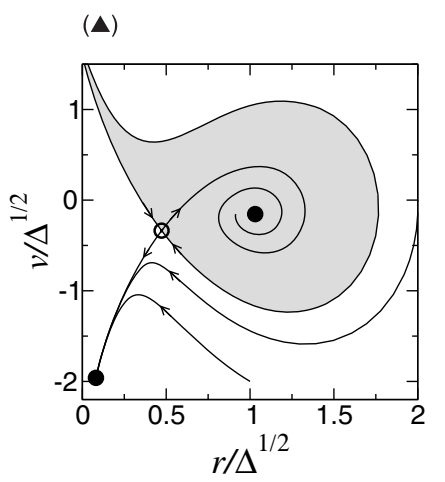

FIG. 1. Analysis of the steady states of FREs (12). (a) Phase diagram: In the wedge-shaped cyan-shaded region there is bistability between a high- and a low-activity state. The boundary of the bistability region is the locus of a saddle-node bifurcation which is exactly obtained in parametric form: $(\bar{\eta}, J)_{\mathrm{SN}}=\left[-\pi^{2} r^{2}-3 \Delta^{2} /(2 \pi r)^{2}, 2 \pi^{2} r+\Delta^{2} /\left(2 \pi^{2} r^{3}\right)\right]$. To the right of the dashed line, defined by $\bar{\eta}_{f}=-[J /(2 \pi)]^{2}-(\pi \Delta / J)^{2}$, there is a stable focus (shaded regions). (b) $r-\bar{\eta}$ and (c) $v-\bar{\eta}$ bifurcation diagrams for $J / \Delta^{1 / 2}=15$. Square symbols: Results obtained from numerical simulations of QIF neurons (see Appendix A for details). (d) Phase portrait of the system in the bistable region $\left[\bar{\eta} / \Delta=-5, J / \Delta^{1 / 2}=15\right.$, triangle in (a)] with three fixed points: a stable focus (with its basin of attraction shaded), a stable node, and a saddle point. 

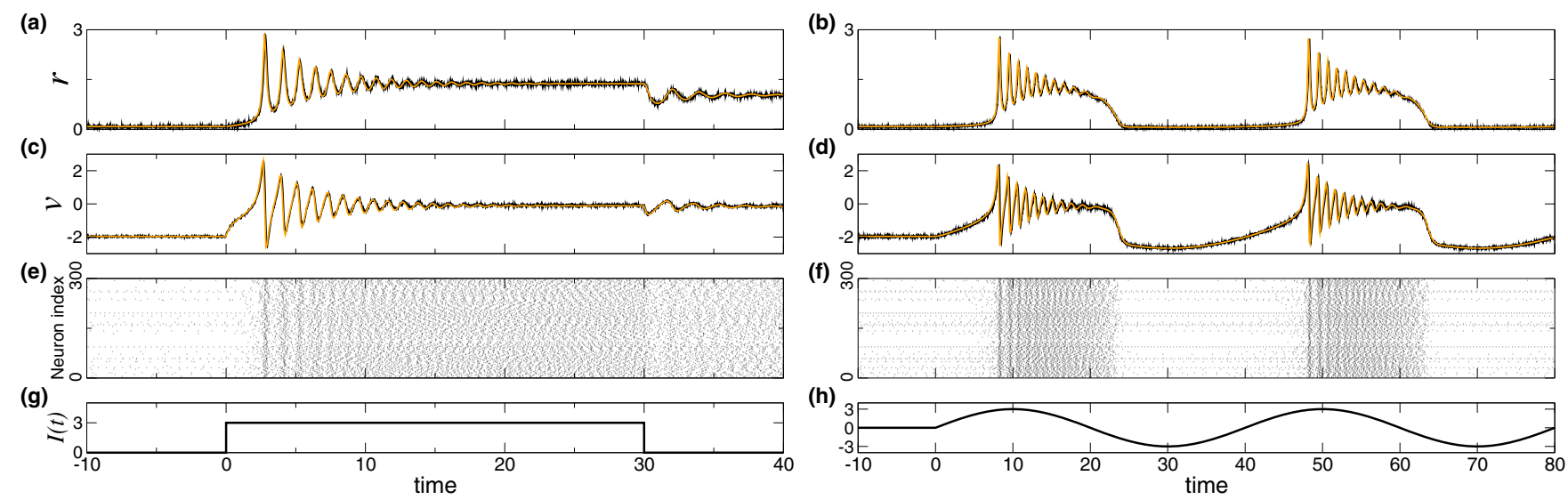

FIG. 2. The transient dynamics of an ensemble of QIF model neurons [Eqs. (1) and (2)] are exactly described by the FREs (12). (a),(b) Instantaneous firing rate and (c),(d) mean membrane potential of the QIF neurons and the FREs are depicted in black and orange, respectively. (e),(f) Raster plots of 300 randomly selected QIF neurons of the $N=10^{4}$ in the ensemble. (a),(c),(e),(g) At time $t=0$, a current $I=3$ is applied to all neurons, and set to zero again at $t=30$; stimulus $I(t)$ shown in (g). (b),(d),(f),(h) At time $t=0$, a sinusoidal current is applied to all neurons $I(t)=I_{0} \sin (\omega t)$, with $I_{0}=3, \omega=\pi / 20$; stimulus $I(t)$ shown in (h). Parameters: $J=15, \bar{\eta}=-5, \Delta=1$.

observe periodic bursting behavior when the system visits the stable focus region of the phase diagram, Fig. 1(a).

To further illustrate the potential of the FREs (12) to predict and investigate complex dynamics in ensembles of spiking neurons, we present here a simple situation where the system of QIF neurons exhibits macroscopic chaos. This is observed by increasing the frequency $\omega$ of the sinusoidal driving, so that the system cannot trivially follow the stable fixed point at each cycle of the applied current.

Figure 3(a) shows a phase diagram obtained using Eq. (12). The shaded regions indicate parameter values where the rate model has either a single chaotic attractor (in black) or a chaotic attractor coexisting with a periodic orbit (in cyan). A trajectory on the chaotic attractor is depicted in Fig. 3(b), and the clearly irregular time series of the firing rate is shown in orange in Fig. 3(d). Using the same parameters, we perform numerical simulations of the QIF neurons [Eqs. (1) and (2)], finding a similar attractor and irregular dynamics as in the rate model; see Figs. 3(c) and 3(d). To obtain the time series shown in Fig. 3(d), we run the QIF neurons numerically and, after a long transient, at time $t=0$, the rate model Eq. (12) is initiated with the values of $r$ and $v$ obtained from the population of QIF neurons. The time series of the two systems, which are initially close, rapidly diverge, reflecting the chaotic nature of the system.

Finally, to illustrate this chaotic state at the microscopic level, Fig. 3(e) shows the raster plot for 300 randomly chosen neurons, corresponding to the time series in Fig. 3(d). The irregular firing of neurons in Fig. 3(e) has some underlying structure that may be visualized ordering the same set of neurons according to their intrinsic currents as $\eta_{k}<\eta_{k+1}$, with $k \in[1,300]$; see Fig. 3(f). Clearly, the maxima of the firing rate coincide with the synchronous firings of clusters of neurons with similar $\eta$ values. The size of these clusters is highly irregular in time, in concomitance with the chaotic behavior.

\section{E. Validity of the Lorentzian ansatz}

Thus far, we have shown that the LA Eq. (5) solves the continuity equation (4) and confirmed that these solutions agree with the numerical simulations of the original system of QIF neurons [Eqs. (1) and (2)]. Here, we further clarify why the LA holds for ensembles of QIF neurons.

Transforming the voltage of the QIF neuron into a phase via $V_{j}=\tan \left(\theta_{j} / 2\right)$, the system [Eqs. (1) and (2)] becomes an ensemble of "theta neurons" [38]:

$$
\dot{\theta}_{j}=\left(1-\cos \theta_{j}\right)+\left(1+\cos \theta_{j}\right)\left[\eta_{j}+J s(t)+I(t)\right] .
$$

In the new phase variable $\theta \in[0,2 \pi)$, the LA Eq. (5) becomes

$$
\tilde{\rho}(\theta \mid \eta, t)=\frac{1}{2 \pi} \operatorname{Re}\left[\frac{1+\alpha(\eta, t) e^{i \theta}}{1-\alpha(\eta, t) e^{i \theta}}\right],
$$

where the function $\alpha(\eta, t)$ is related to $w(\eta, t)$ as

$$
\alpha(\eta, t)=\frac{1-w(\eta, t)}{1+w(\eta, t)} .
$$

Equations (5) and (14) are two representations of the socalled Poisson kernel on the half-plane and on the unit disk, respectively. These representations are related via Eq. (15), which establishes a conformal mapping from the half-plane $\operatorname{Re}(w) \geq 0$ onto the unit disk $|\alpha| \leq 1$. In the next section, we show that variables $r$ and $v$ can be related, via the same conformal map, to the Kuramoto order parameter, which is a macroscopic measure of phase coherence $[43,44]$. 
(a)

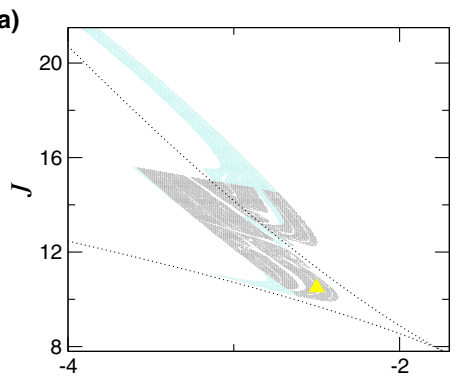

(d)

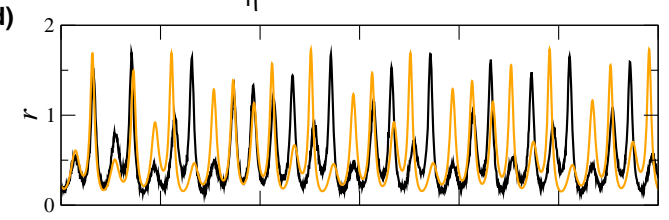

(e)

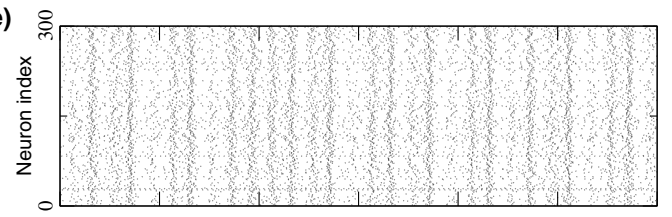

(f) $\times$

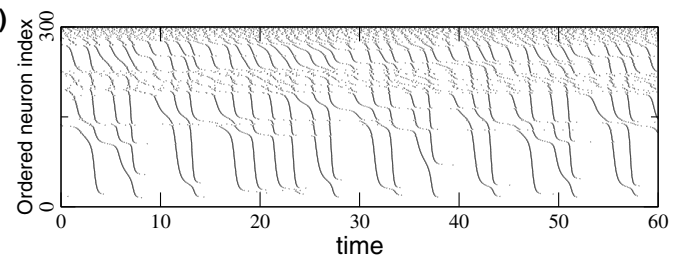

FIG. 3. Firing-rate model predicts the existence of macroscopic chaos in ensemble QIF neurons [Eq. (1)] with an injected periodic current $I(t)=I_{0} \sin (\omega t)$. (a) Phase diagram with the regions where chaos is found, obtained using the rate model Eq. (12). In the black-shaded region, there is only one chaotic attractor, whereas in the cyan region the chaotic attractor coexists with a periodic attractor. Dotted lines correspond to the bistability boundary of Fig. 1(a), depicted to facilitate comparison. (b) Chaotic trajectory obtained simulating the FREs (12); the Lyapunov exponent is $\lambda=0.183 \ldots$. (c) Chaotic trajectory obtained from the QIF neurons-parameters corresponding to the (yellow) triangle symbol in (a). (d) Time series for the rate model (orange) and QIF model (black). (e) Raster plot corresponding to 300 randomly selected neurons. (f) Same raster plot as in (e), ordering neurons according to their intrinsic current $\eta_{k}$. Parameters: $I_{0}=3, \omega=\pi$ and $\bar{\eta}=-2.5, J=10.5$ [triangle symbol in (a)].

The key observation supporting the applicability of the LA is the fact that Eq. (14) turns out to be the ansatz discovered in 2008 by Ott and Antonsen [35]. According to the OA theory, in the thermodynamic limit, the dynamics of the class of systems

$$
\partial_{t} \theta(\eta, t)=\Omega(\eta, t)+\operatorname{Im}\left[H(\eta, t) e^{-i \theta}\right]
$$

generally converges to the OA manifold [Eq. (14)]. In our case, for Eq. (13), we have $\Omega(\eta, t)=1+\eta+J_{s}+I$ and $H(\eta, t)=i\left(-1+\eta+J_{S}+I\right)$. Thus far, the convergence of Eq. (16) to the OA manifold has been only proven for $H(\eta, t)=H(t)$. This includes the well-known Kuramoto

and Winfree models [45,46], but not Eq. (13). However, there are theoretical arguments [47] that strongly suggest that the OA manifold is also attracting for $\eta$-dependent $H$, as numerically confirmed by a number of recent papers using theta neurons [48-50] and other phase-oscillator models [51-55].

\section{F. Firing rate and Kuramoto order parameter}

There exists a mapping between the macroscopic variables $r$ and $v$ and the so-called Kuramoto order parameter $Z$. Equation (15) relates, for each value of $\eta$, the firing rate and the mean membrane potential, both contained in $w$, with the uniformity of the phase density, measured by $\alpha$-note that $\alpha=0$ in Eq. (14) yields a perfectly uniform density. The Kuramoto order parameter is obtained by integrating $\alpha$ over the whole population as

$$
\begin{aligned}
Z(t) & =\int_{-\infty}^{\infty} g(\eta) \int_{0}^{2 \pi} \tilde{\rho}(\theta \mid \eta, t) e^{i \theta} d \theta d \eta \\
& =\int_{-\infty}^{\infty} g(\eta) \alpha^{*}(\eta, t) d \eta,
\end{aligned}
$$

where we assume that the density $\tilde{\rho}$ is the OA ansatz Eq. (14). The quantity $Z$ can be seen as the center of mass of a population of phases distributed across the unit circle $e^{i \theta}$.

For a Lorentzian distribution of currents, $g(\eta)$, we may derive the exact formula (see Appendix B)

$$
Z=\frac{1-W^{*}}{1+W^{*}},
$$

relating the Kuramoto order parameter $Z$ with the firingrate quantity $W \equiv \pi r+i v$. Figure 4 illustrates this conformal mapping of the right half-plane $(r \geq 0)$ onto the unit disk $(|Z| \leq 1)$.

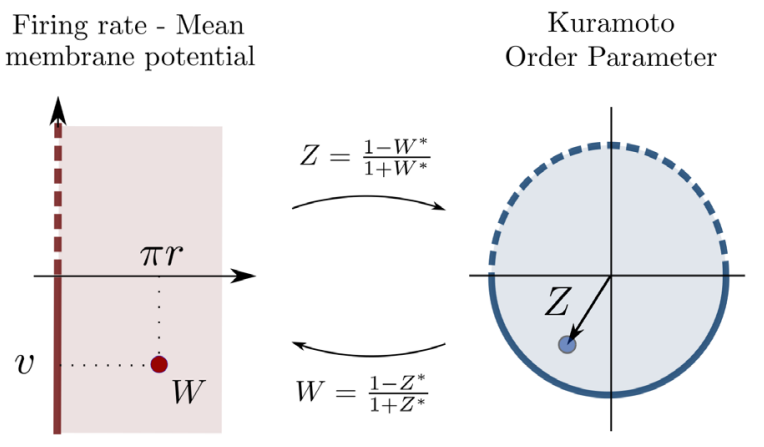

FIG. 4. The conformal map Eq. (18) transforms the right halfplane onto the unit disk. This transformation and its inverse define a one-to-one mapping between the Kuramoto order parameter $Z$ and the macroscopic quantities of the population of QIF neurons: $r$, firing rate; $v$, mean membrane potential. Note that in the limit $Z=e^{i \Psi}$ [full coherence, $\tilde{\rho}(\theta)=\delta(\theta-\Psi)$ ], we recover $v=i\left(1-e^{i \Psi}\right) /\left(1+e^{i \Psi}\right)=\tan (\Psi / 2)$, as the original mapping $V=\tan (\theta / 2)$ between QIF and theta neurons dictates. 


\section{CONCLUSIONS}

We present a method for deriving firing-rate equations for a network of heterogeneous QIF neurons, which is exact in the thermodynamic limit. To our knowledge, the resulting system of ordinary differential equations (12) represents the first exact FREs of a network of spiking neurons.

We emphasize that the derivation of the FREs does not rely on assumptions of weak coupling, separation of time scales, averaging, or any other approximation. Rather, the assumptions underlying the validity of Eq. (12) are that (i) the QIF neurons are all-to-all connected, (ii) heterogeneity is quenched, and (iii) inputs are distributed according to a Lorentzian distribution.

The last two assumptions may seem particularly restrictive. Concerning assumption (iii), it must be stressed that for arbitrary distributions of quenched heterogeneity, the LA Eq. (5) remains generally valid, and, therefore, Eq. (10) can be used to discern the stability of the network states (see Appendix C). Furthermore, in Appendix C, we also show numerical simulations using uniform- and Gaussiandistributed inputs that reveal very similar macroscopic dynamics in response to time-varying inputs. Even relaxing assumption (ii), numerical simulations with identical QIF neurons driven by external independent Gaussian noise sources show qualitative agreement with the FREs (12) (see Appendix D). In summary, the choice of a quenched Lorentzian distribution $g(\eta)$ is, thus, a mere mathematical convenience, whereas the insights gained from the resulting firing-rate description are valid more generally.

Our derivation represents a sharp departure from and a major advance compared to previous studies in several regards. First, in the past it has been possible to calculate only the approximate firing rate of networks of spiking neurons for stationary states, or for weak deviations of such states $[9-12,15,19,20]$. The equations that result from these derivations are difficult to solve, and typically require special numerical methods. In contrast, FREs (12) can be easily analyzed and simulated, and exactly reproduce the behavior of the spiking network far from any fixed point and for arbitrary external currents. Second, firing-rate descriptions traditionally assume that the activity of a population of neurons is equivalent to a set of uncorrelated stochastic processes with a given rate; see, e.g., Refs. [13,14]. However, in simulations of spiking networks, it is well known that the response of the network to nonstationary inputs generally involves some degree of spike synchrony. Recent theoretical work has sought to improve on classical rate models, which act as a low-pass filter of inputs, by fitting them with linear filters extracted from the corresponding Fokker-Planck equation for the network $[19,20]$. These filters tend to generate damped oscillations when the external noise level is not too high, reflecting the presence of spike synchrony $[15,20]$. The FREs (12) also capture this phenomenon and reveal that the underlying mechanism is an interplay between firing rate and subthreshold voltage. Furthermore, because these equations are exact, we can explore the full nonlinear response of the network, such as the generation of chaotic states of synchronous bursting, as shown in Fig. 3.

Recently, it has been shown that not only Kuramoto-like models, but a much wider class of phase models, evolve in the OA manifold defined by Eq. (14). Specifically, networks of pulse-coupled oscillators [46] and theta neurons [48-50] allow for an exact, low-dimensional description in terms of the Kuramoto order parameter [Eq. (17)]. Although these later works use a finite-width phasecoupling function that differs from the synaptic coupling [Eq. (3)], the obtained low-dimensional description in terms of the Kuramoto order parameter is analogous to ours, but in a different space [56]. Indeed, we show that the $\mathrm{OA}$ ansatz Eq. (14) is related, via the nonlinear transformation of variables Eq. (15), to the LA ansatz Eq. (5). Remarkably, this transformation establishes an exact correspondence between the Kuramoto order parameter and a novel, biophysically meaningful macroscopic observable that describes the firing rate and mean membrane potential of the neuronal network. Interestingly, the low-dimensional description in terms of firing rates seems to be a more natural description for networks of spiking neurons, compared to that in terms of the Kuramoto order parameter. The firing-rate equations (12) take a surprisingly simple form in the LA manifold, which makes them a valuable tool to explore and understand the mechanisms governing the macroscopic dynamics of neuronal networks.

Finally, since the OA ansatz is the asymptotic solution for systems of the form in Eq. (16), applying the change of variables $V=\tan (\theta / 2)$ automatically implies that the LA should hold for populations governed by

$$
\dot{V}_{j}=A\left(\eta_{j}, t\right)+B\left(\eta_{j}, t\right) V_{j}+C\left(\eta_{j}, t\right) V_{j}^{2},
$$

where $A, B$, and $C$ are related to $\Omega$ and $H$ as $A=[\Omega+\operatorname{Im}(H)] / 2, B=-\operatorname{Re}(H), C=[\Omega-\operatorname{Im}(H)] / 2$. Notably, Eq. (19) defines a wide family of ensembles of QIF neurons.

Therefore, the LA is actually valid for more general networks beyond the one investigated here $-\eta_{j}$ in Eq. (19) may also be a vector containing several forms of disorder. As particularly relevant cases, in Appendix E, we provide the FREs governing the dynamics of an excitatory network with both heterogeneous inputs $\eta$ and synaptic weights $J$, as well as a pair of interacting excitatory and inhibitory populations of QIF neurons. In addition, according to Eq. (19), the LA is also valid if synapses are modeled as conductances, in which case the reversal potentials may be distributed as well. Moreover, the role of gap junctions or synaptic kinetics may be considered within the same framework. 


\section{ACKNOWLEDGMENTS}

D. P. and A.R. acknowledge support by MINECO (Spain) under the Ramón y Cajal program. E. M. and A. R. acknowledge support from a grants from the Spanish Ministry of Economics and Competitiveness PSI201342091 and BFU2012-33413.

\section{APPENDIX A: NUMERICAL SIMULATIONS}

To numerically simulate the population of QIF neurons [Eq. (1)], we use the Euler method, with time step $d t=10^{-4}$. The population has $N=10^{4}$ neurons, and the Lorentzian distribution Eq. (11) is deterministically generated using $\eta_{j}=\bar{\eta}+\Delta \arctan [\pi / 2(2 j-N-1) /(N+1)]$, where $j=1, \ldots, N$ and $\Delta=1$.

The time it takes for the membrane potential of a QIF neuron (with $I_{j}>0$ ) to reach infinity from a given positive value of the membrane potential is $\arctan \left(\sqrt{I_{j}} / V_{p}\right) / \sqrt{I_{j}}$. For $\sqrt{I_{j}} \ll V_{p}$, this expression can be approximated as

$$
\frac{\arctan \left(\sqrt{I_{j}} / V_{p}\right)}{\sqrt{I_{j}}} \approx \frac{1}{V_{p}} .
$$

In simulations, we consider $V_{p}=-V_{r}=100$, and use the previous approximation. Thus, the time for the neurons to reach infinity from $V_{p}$ is $1 / V_{p} \approx 10^{-2}$. Additionally, the time taken from minus infinity to $V_{r}$ is $1 / V_{r} \approx 10^{-2}$. Numerically, once a neuron's membrane potential $V_{j}$ satisfies $V_{j} \geq V_{p}$ the neuron is reset to $-V_{j}$ and held there for a refractory time $2 / V_{j}$. The neurons produce a spike when $V_{j}$ reaches infinity, i.e., a time interval $1 / V_{j}$ after crossing $V_{p}$. The exact time of the spike cannot be evaluated exactly in numerical simulations, since $d t$ is finite. However, simulations agree very well with the theory, provided that $1 / V_{p} \gg d t$.

To evaluate the mean membrane potential $v$, the population average is computed discarding those neurons in the refractory period. The choice $V_{p}=-V_{r}$ is not needed, but in this way the observed mean membrane potential $v$ agrees with the theory, consistent with the definition in Eq. (8). The mean synaptic activation Eq. (3) is evaluated using the Heaviside step function $a_{\tau}(t)=\Theta(\tau-t) / \tau$, with $\tau=10^{-3}$. The instantaneous firing rates are obtained binning time and counting spikes within a sliding time window of size $\delta t=2 \times 10^{-2}$ (Figs. 2,6-8) and $\delta t=4 \times 10^{-2}$ (Fig. 3).

\section{APPENDIX B: PROOF OF EQ. (18)}

The inverse of Eq. (18) reads

$$
W=\frac{1-Z^{*}}{1+Z^{*}} .
$$

Recalling Eqs. (7) and (9), we write the macroscopic field $W$ as

$$
W(t)=\int_{-\infty}^{\infty} w(\eta, t) g(\eta) d \eta
$$

where $w \equiv x+i y$. Inserting the conformal mapping $w=(1-\alpha) /(1+\alpha)$ - the inverse of Eq. (15)—we get

$$
W(t)=\int_{-\infty}^{\infty}\left[\frac{1-\alpha(\eta, t)}{1+\alpha(\eta, t)}\right] g(\eta) d \eta .
$$

Using the geometric series formula, and grouping the powers of $\alpha$, we may evaluate the integrals, obtaining

$$
W(t)=1-2 Z^{*}(t)+2 Z_{2}^{*}(t)-2 Z_{3}^{*}(t)+\cdots,
$$

where the $Z_{m}$ are the generalized order parameters [57]:

$$
\begin{aligned}
Z_{m}(t) & =\int_{-\infty}^{\infty} g(\eta) \int_{0}^{2 \pi} \tilde{\rho}(\theta \mid \eta, t) e^{i m \theta} d \theta d \eta \\
& =\int_{-\infty}^{\infty} g(\eta)\left[\alpha^{*}(\eta, t)\right]^{m} d \eta .
\end{aligned}
$$

Since, for Lorentzian $g(\eta), Z_{m}(t)=\left[\alpha^{*}(\eta=\bar{\eta}-i \Delta, t)\right]^{m}=$ $Z(t)^{m}$, we can revert the power series in Eq. (B2) and obtain the result in Eq. (B1).

\section{APPENDIX C: RESULTS FOR ARBITRARY DISTRIBUTIONS OF CURRENTS}

In this Appendix, we compare the results in the main text, obtained using a Lorentzian distribution of currents $g(\eta)$, with other distributions. The results are qualitatively similar, as evidenced by Figs. 5-7 (cf. Figs. 1-3).

Note that, if a particular distribution $g(\eta)$ has $2 n$ poles (all of them off the real axis). one can readily obtain the FREs consisting of $n$ complex-valued ordinary differential equations. Even if $g(\eta)$ does not fulfill this condition, as in the case of Gaussian or uniform distributions, the Lorentzian ansatz still holds (see below).

Moreover, it is possible to efficiently simulate the dynamics of a population by integrating Eq. (10) for a sample of $\eta$ values that approximate a particular $g(\eta)$ [58]. However, if $g(\eta)$ is a discrete distribution (or has a discrete part), as in the case of the Dirac delta function $g(\eta)=\delta(\eta-\bar{\eta})$, the so-called Watanabe-Strogatz theory applies $[47,60,61]$, and the "Lorentzian manifold" is no longer attracting since the dynamics is extremely degenerate, akin to a Hamiltonian system.

\section{Steady states}

Considering $I(t)=0$ in Eqs. (1) and (2), it is possible to find the solutions with steady firing rate $r(t)=r_{0}$ for arbitrary distributions of currents. 

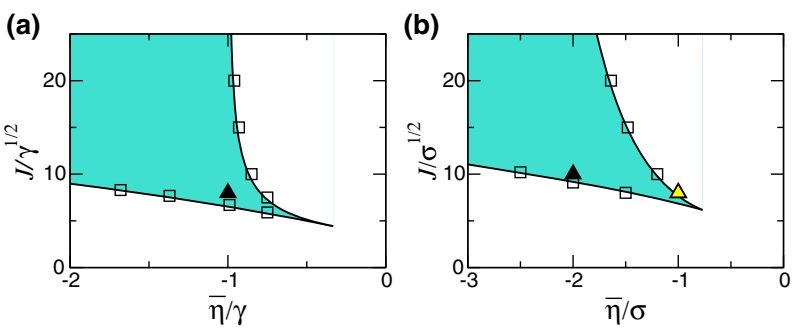

FIG. 5. Phase diagrams for (a) uniform and (b) Gaussian distributions of currents $g(\eta)$. The cyan-shaded region represents the bistable region, with the solid lines corresponding to saddlenode bifurcations analytically obtained from Eqs. (C5) and (C6). Square symbols are estimates of the bifurcations' loci obtained by direct numerical simulations of $N=10^{4}$ QIF neurons. Triangle symbols indicate parameter values used in numerical simulations of Figs. 6 and 7.

As we point out in the main text, the stationary solution of the continuity equation (4) for those neurons that are intrinsically active $\left(\eta>-J r_{0}\right)$ must be inversely proportional to their speed; that is,

$$
\rho_{0}(V \mid \eta)=\frac{c(\eta)}{V^{2}+\eta+J r_{0}}
$$

where the normalizing constant is $c(\eta)=\sqrt{\eta+J r_{0}} / \pi$. The firing rate for each value of $\eta$ is then

$$
r_{0}(\eta)=\rho_{0}(V \rightarrow \infty \mid \eta) \dot{V}(V \rightarrow \infty \mid \eta, t)=c(\eta),
$$

and integration over all the firing neurons gives the self-consistent condition for $r_{0}$ :

$$
r_{0}=\int_{-J r_{0}}^{\infty} c(\eta) g(\eta) d \eta
$$

(a)

(b)

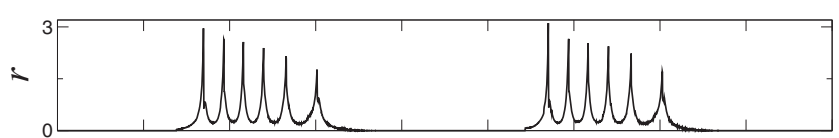

)

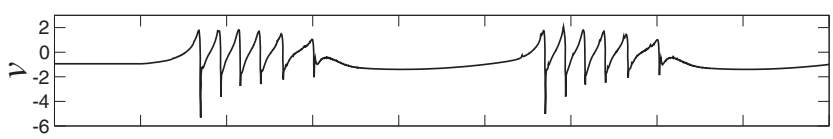

(c)

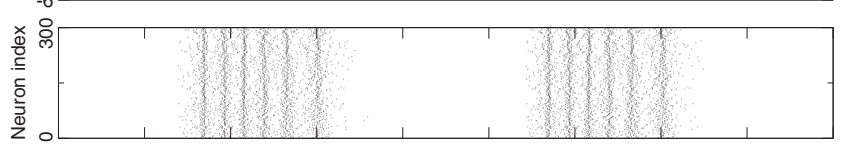

(d)

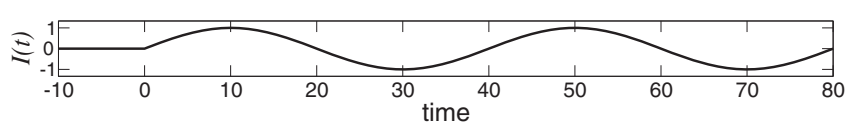

which is valid for any distribution $g(\eta)$ [an analogous expression was obtained in Eq. (45) of Ref. [40]]. For Lorentzian $g(\eta)$, the integral in Eq. (C1) can be evaluated, and solving the resulting equation for $r_{0}$, one obtains the steady states in agreement with the result of setting $\dot{r}=\dot{v}=0$ in the FREs (12).

\section{Linear stability analysis of steady states}

Assuming that the LA captures the actual dynamics of the population of QIF neurons, one can also investigate the stability of steady states for arbitrary distributions $g(\eta)$. Indeed, the evolution of infinitesimal perturbations from a steady state,

$w_{0}(\eta) \equiv x_{0}(\eta)+i y_{0}(\eta)= \begin{cases}\sqrt{\eta+J r_{0}} & \text { if } \eta>-J r_{0} \\ -i \sqrt{-\eta-J r_{0}} & \text { if } \eta \leq-J r_{0}\end{cases}$

is determined by the linearization of Eq. (10):

$$
\partial_{t} \delta w(\eta, t)=i\left(J \delta r-2 w_{0} \delta w\right),
$$

where $\delta r(t)=(2 \pi)^{-1} \int_{-\infty}^{\infty}\left[\delta w(\eta, t)+\delta w^{*}(\eta, t)\right] g(\eta) d \eta$.

To find the discrete spectrum of eigenvalues [62], we use the ansatz $\delta w(\eta, t)=b(\eta) e^{\lambda t}$ in Eq. (C2) and obtain

$\left[\lambda+2 w_{0}(\eta) i\right] b(\eta) e^{\lambda t}=\frac{i J}{2 \pi} \int_{-\infty}^{\infty}\left[b(\eta) e^{\lambda t}+b^{*}(\eta) e^{\lambda^{*} t}\right] g(\eta) d \eta$.

Solving the equation for $b e^{\lambda t}$, we find

$b(\eta) e^{\lambda t}=\frac{1}{2 w_{0}(\eta)-i \lambda} \frac{J}{2 \pi} \int_{-\infty}^{\infty}\left[b(\eta) e^{\lambda t}+b^{*}(\eta) e^{\lambda^{*} t}\right] g(\eta) d \eta$.

After summing the complex conjugate at each side of the equation, we multiply by $g(\eta)$ and integrate over $\eta$. This

(e)
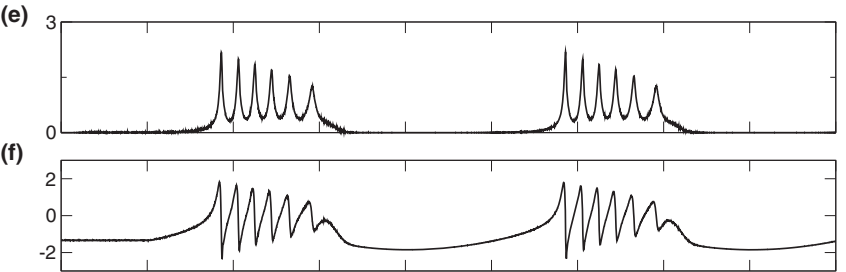

(g)

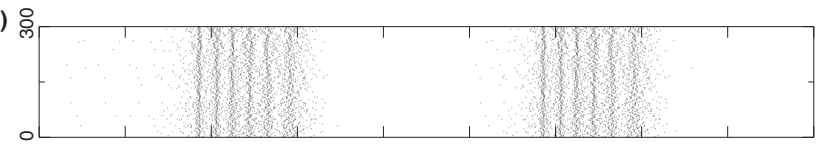

(h)

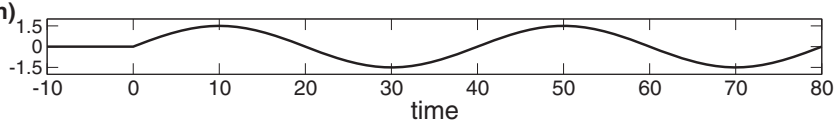

FIG. 6. Numerical simulations of the excitatory network of QIF neurons, Eqs. (1) and (2). with (a)-(d) uniform and (e)-(h) Gaussian distributions of currents. As in Fig. 2, at time $t=0$, an external sinusoidal current $I(t)=I_{0} \sin (\omega t)$ —shown in (d) and (h) —is applied to all neurons. (a),(e) Time series of the firing rate and (b),(f) the mean membrane potential of all cells. In (c) and (g), we depict the raster plots of 300 randomly chosen neurons. Parameters correspond to the black triangle symbols in Fig. 5: (a) - (d) $\bar{\eta}=-1, J=8, \gamma=1$, $I_{0}=1$; (e)-(h) $\bar{\eta}=-2, J=10, \sigma=1, I_{0}=1.5$. 
(a)

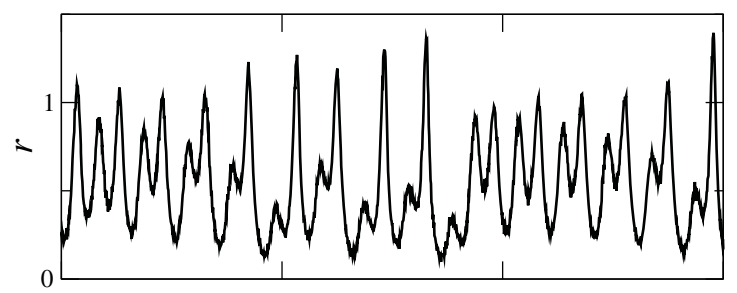

(b)

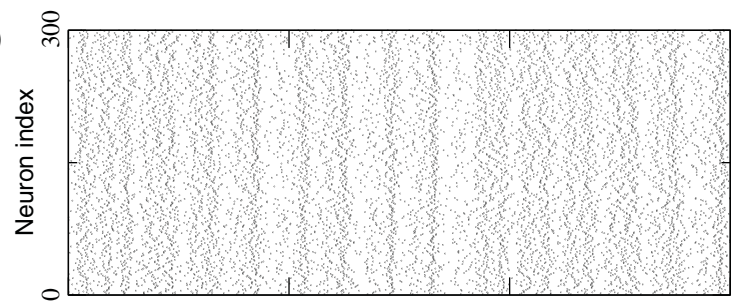

(c)

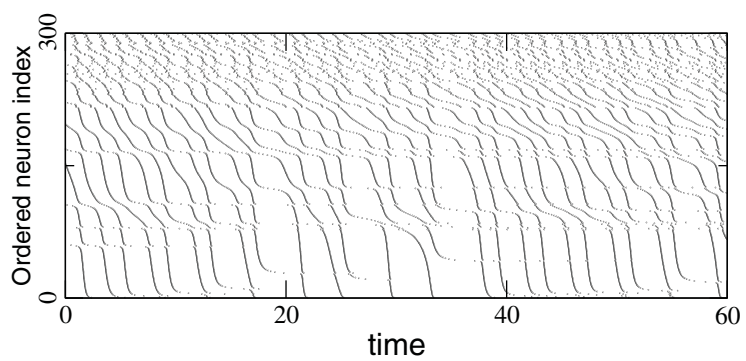

FIG. 7. Chaotic state in a network of $N=10^{4}$ QIF neurons with Gaussian-distributed currents. Network parameters correspond to the yellow triangle in the phase diagram of Fig. 5(b). As in Fig. 3, neurons receive a common periodic current $I(t)=I_{0} \sin (\omega t)$ of frequency $\omega=\pi$. Parameters: $\bar{\eta}=-1, J=8, \sigma=1, I_{0}=1.5$.

allows one to cancel out the integrals $\int\left(b e^{\lambda t}+b^{*} e^{\lambda^{*} t}\right) g d \eta$. Then, imposing self-consistency, we find

$$
1=\frac{J}{2 \pi} \int_{-\infty}^{\infty}\left[\frac{1}{2 w_{0}(\eta)-i \lambda}+\text { c.c. }\right] g(\eta) d \eta \text {. }
$$

Local bifurcations are associated with marginal stability of the fixed points: $\operatorname{Re}(\lambda) \rightarrow 0$. In correspondence with the results obtained for a Lorentzian distribution in the main text, we expect bifurcations of saddle-node type associated with $\lambda=0$. Therefore, from Eq. (C3) we find that the loci of the saddle-node bifurcations are located at

$$
J_{\mathrm{SN}}=\frac{2 \pi}{\int_{-\infty}^{\infty} \frac{x_{0}(\eta)}{\left|w_{0}(\eta)\right|^{2}} g(\eta) d \eta} .
$$

Since $x_{0}=\sqrt{\eta+J r_{0}}$ for $\eta>-J r_{0}$, and zero otherwise, we can restrict the integration over $\eta$ to the range $\left(-J r_{0}, \infty\right)$. Now, multiplying Eq. (C4) by Eq. (C1), we get, after defining $\xi=J_{\mathrm{SN}} r_{0}$, two equations that permit us to find the locus of the saddle-node bifurcations systematically for arbitrary distributions of currents:

$$
J_{\mathrm{SN}}=\frac{2 \pi}{\int_{0}^{\infty} \eta^{-1 / 2} g(\eta-\xi) d \eta},
$$

with $\xi$ obtained solving

$$
\xi=\frac{2 \int_{0}^{\infty} \eta^{1 / 2} g(\eta-\xi) d \eta}{\int_{0}^{\infty} \eta^{-1 / 2} g(\eta-\xi) d \eta}
$$

\section{Bistability region for uniform and Gaussian distributions}

Equations (C5) and (C6) are particularly easy to solve for uniform distributions of currents:

$$
g(\eta)= \begin{cases}\frac{1}{2 \gamma} & \text { for }|\eta-\bar{\eta}|<\gamma \\ 0 & \text { otherwise }\end{cases}
$$

After defining the rescaled parameters $\tilde{\eta}=\bar{\eta} / \gamma$ and $\tilde{J}=J / \sqrt{\gamma}$, we find two branches of saddle-node bifurcations emanating from the cusp point at $\tilde{\eta}=-1 / 3$ :

$$
\begin{aligned}
& \tilde{J}_{\mathrm{SN}}^{(1)}=\frac{2 \pi}{\sqrt{3 \tilde{\eta}+3}}, \\
& \tilde{J}_{\mathrm{SN}}^{(2)}=\frac{2 \pi}{\sqrt{\tilde{\eta}+1+2 \sqrt{\frac{1}{3}+\tilde{\eta}^{2}}}-\sqrt{\tilde{\eta}-1+2 \sqrt{\frac{1}{3}+\tilde{\eta}^{2}}}} .
\end{aligned}
$$

These functions are plotted in Fig. 5(b). Numerical simulations using the network of QIF neurons confirm the correctness of these boundaries (see the square symbols in Fig. 5).

For Gaussian distributions,

$$
g(\eta)=\frac{1}{\sqrt{2 \pi} \sigma} e^{-(\eta-\bar{\eta})^{2} /\left(2 \sigma^{2}\right)},
$$

the solutions of Eqs. (C5) and (C6) can be numerically found. The results are shown in Fig. 5(b). Again, there is a perfect agreement between Eqs. (C5) and (C6) - derived assuming the Lorentzian Ansatz-and the numerical estimations obtained simulating the network of QIF neurons.

\section{Excitatory networks with external periodic currents}

Firing-rate equations (12) predict the existence of a stable focus in the shaded region of Fig. 1. Trajectories attracted to this fixed point display oscillations in the firing rate and mean membrane potential due to the transient synchronous firing of the QIF neurons.

When an external periodic current is injected to all neurons in the network, the spiral dynamics around the fixed point is responsible for the bursting behavior observed in Fig. 2, as well as for the emergence of the macroscopic chaos shown in Fig. 3. Here, we investigate whether similar phenomena occur when an external 
periodic current of the same frequency is injected in an excitatory network with either uniform or Gaussiandistributed currents.

We introduce a sinusoidal forcing $I(t)=I_{0} \sin (\omega t)$ observing a behavior qualitatively identical to the one reported in the main text with Lorentzian $g(\eta)$. Under a low-frequency forcing, the system displays periodic bursting, see Fig. 6, provided the parameters are set inside the bistable region (see the black triangles in Fig. 5). Furthermore, note that the range of firing rates and mean membrane potentials in Fig. 6 are similar to those of Fig. 2-for Lorentzian distributions of currents.

As for the simulations shown in Fig. 3, we next increase the frequency of the injected current up to $\omega=\pi$ to investigate the emergence of macroscopic chaos in a network with Gaussian-distributed currents. Figure. 7 shows the emergence of an apparently chaotic state. The observed dynamics is similar to that of Fig. 3, which suggests it is chaotic. This type of chaos persists in the thermodynamic limit $N \rightarrow \infty$. On top of this, highly dimensional but weakly chaotic dynamics is probably also present due to "residual" finite-size effects.

\section{APPENDIX D: IDENTICAL QIF NEURONS DRIVEN BY INDEPENDENT NOISE TERMS}

Now, we compare the results obtained above and in the main text for quenched heterogeneity $g(\eta)$ with the results for identical neurons $g(\eta)=\delta(\eta-\bar{\eta})$ driven by noise. Specifically, the input currents are now taken as

$$
I_{j}=\bar{\eta}+J r(t)+\xi_{j}(t),
$$

where $\xi_{j}$ are independent white noise terms with expected values $\left\langle\xi_{j}(t)\right\rangle=0$, and $\left\langle\xi_{j}(t) \xi_{k}\left(t^{\prime}\right)\right\rangle=2 D \delta_{j k} \delta\left(t-t^{\prime}\right)$.

In the thermodynamic limit, the density $\rho(V, t)$ obeys the Fokker-Planck equation:

$$
\partial_{t} \rho+\partial_{V}\left[\left(V^{2}+\bar{\eta}+J r\right) \rho\right]=D \partial_{V}^{2} \rho .
$$

The Lorentzian ansatz is not a solution of this equation, but we demonstrate here that the phenomena observed with quenched heterogeneity also arise with independent noise sources. This qualitative similarity at the macroscopic level between quenched Lorentzian heterogeneity and Gaussian noise has been noted in previous work [63].

A subtle point in Eq. (D2) is that its nondimensionalization entails a different (compared to $\Delta$ ) rescaling with $D$ : $\tilde{V}=V / D^{1 / 3}, \tilde{\eta}=\eta / D^{2 / 3}, \tilde{J}=J / D^{1 / 3}, \tilde{t}=t D^{1 / 3}$ (implying $\left.\tilde{r}=r / D^{1 / 3}\right)$.

Numerical simulations reveal the existence of a region of bistability, see Fig. 8(a), analogous to the one observed for quenched heterogeneity, cf. Figs. 1(a) and 5. Obviously, true bistability holds only in the thermodynamic limit, while what we observe are rather exceedingly long residence times close to each fixed point (see Fig. 8 caption for details).

Additionally, in order to investigate and compare the dynamical behavior of the identical noise-driven neurons with that of the FREs (12), an external current of intensity
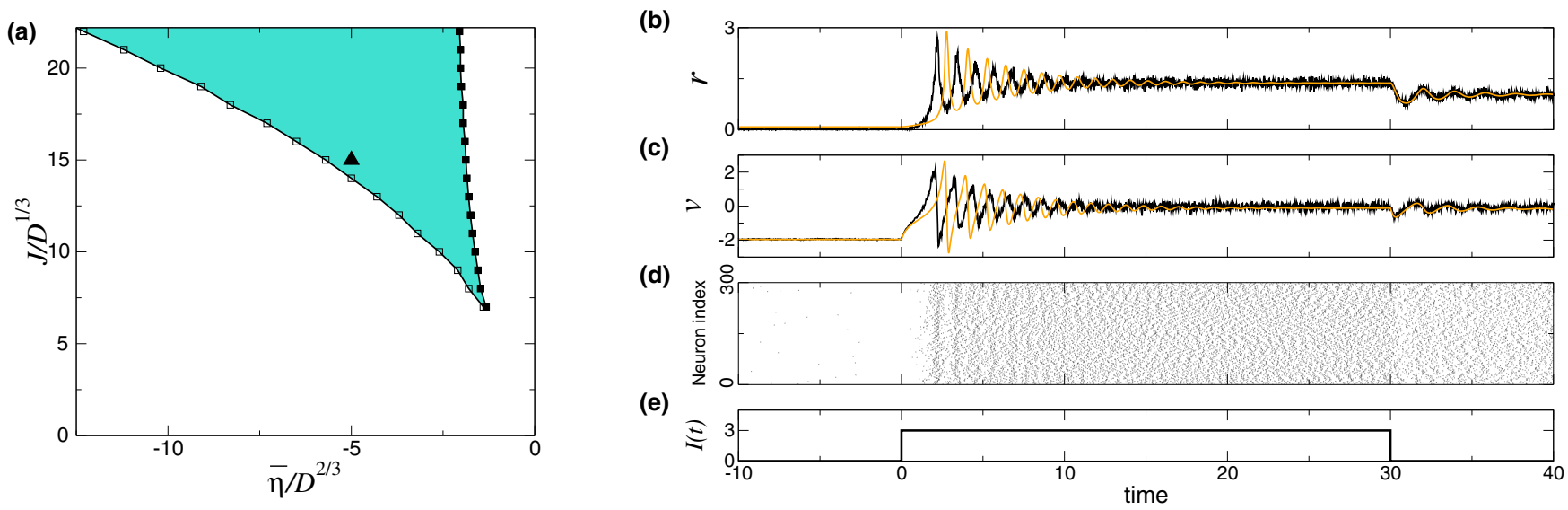

FIG. 8. Numerically obtained phase diagram (a) and time series (b)-(d) for a network of identical QIF neurons driven by independent white noise terms. The square symbols in (a) represent the boundaries that enclose the region of bistability between a high- and a lowactivity state. The boundaries have been obtained using a network of $10^{4}$ neurons, and by numerical continuation of a low-activity solution (filled squares) and a high-activity solution (empty squares). Specifically, using a noise intensity $D=1$ and a particular value of $J$, the system is initialized either at $\bar{\eta}=-5$ or at $\bar{\eta}=0$ and, after $t=10$ time units, parameter $\bar{\eta}$ is decreased or increased an amount 0.025 or 0.1 , respectively. This continuation is made until the relative change of two successive values of the averaged firing rate (time averaged over the last time unit for each parameter value) is larger than 50\%. Other parameters for the numerical simulations are the same as in all other figures, and are described in Appendix A. The triangle symbol indicates the parameter value, $\left(\bar{\eta} / D^{2 / 3}, J / D^{1 / 3}\right)=(-5,15)$, corresponding to the numerical simulations shown in black in the right-hand panels-for the simulations. we use $D=1$. To facilitate the comparison with the FREs (12), the orange curves show the time series of the FREs, using the same parameters $(\bar{\eta}, J)=(-5,15)$, but with $\Delta=1$. 
$I_{0}=3$ is injected to all neurons at time $t=0$, like in Fig. 2 .

In Figs. 8(b) and 8(c), the time series of the firing rate and the mean membrane potential clearly display damped oscillations after the injection of the current, confirming the existence of a stable focus, exactly as observed in the FREs (12). The existence of a stable focus reflects the presence of transient spike synchrony in the network, as seen by the raster plot in Fig. 8(d). Remarkably, the raster plot and the time series closely resemble those of Fig. 2. The resemblance is not just qualitative, but rather there is a near quantitative fit between the network of QIF neurons driven by Gaussian noise and the FREs (12), which are derived assuming quenched Lorentzian heterogeneity (orange curves).

\section{APPENDIX E: MODEL GENERALIZATIONS}

To illustrate the potential of the LA for investigating more sophisticated networks, here we provide the lowdimensional FREs corresponding to an excitatory network of QIF neurons with independently distributed currents and synaptic weights and to two interacting populations of excitatory and inhibitory QIF neurons with distributed currents.

\section{Excitatory population with heterogeneous currents and synaptic weights}

As a first example, let us assume that both the currents $\eta$ and the synaptic weights $J$ are distributed-this type of heterogeneity was also considered in Ref. [40]. The input currents then read [66]

$$
I_{j}=\eta_{j}+J_{j} r(t)+I(t) .
$$

For simplicity, we additionally assume that $\eta$ and $J$ are distributed independently, with a joint distribution $p(\eta, J)=g(\eta) h(J)$. In the simplest situation of Lorentzian $g(\eta)$ and $h(J)$,

$$
g(\eta)=\frac{\Delta / \pi}{(\eta-\bar{\eta})^{2}+\Delta^{2}}, \quad h(J)=\frac{\Gamma / \pi}{(J-\bar{J})^{2}+\Gamma^{2}},
$$

the problem is extremely simplified using the Lorentzian ansatz, which now trivially reads

$$
\rho(V \mid \eta, J, t)=\frac{1}{\pi} \frac{x(\eta, J, t)}{[V-y(\eta, J, t)]^{2}+x(\eta, J, t)^{2}} .
$$

The firing rate and mean membrane potential are determined only by the value of $w \equiv x+i y$ at the poles in the lower half-planes:

$$
\begin{aligned}
r(t)+i v(t) & =\iint w(\eta, J, t) g(\eta) h(J) d \eta d J \\
& =w(\bar{\eta}-i \Delta, \bar{J}-i \Gamma, t) .
\end{aligned}
$$

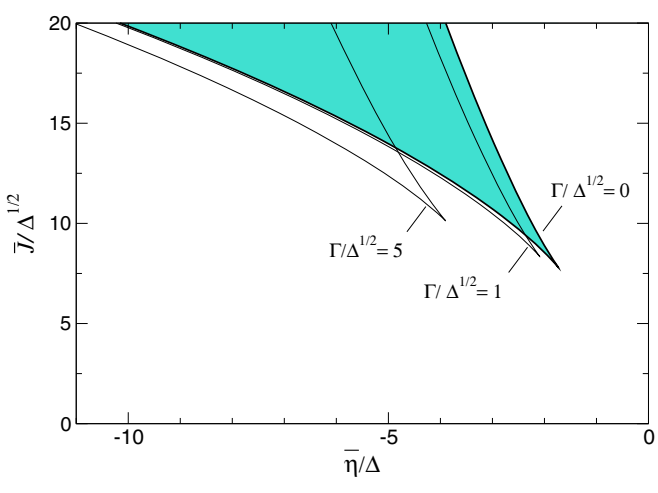

FIG. 9. Phase diagram for an excitatory population with heterogeneous currents and synaptic weights obtained using the FREs (E1).

Finally, evaluating Eq. (10) at the poles $(\eta=\bar{\eta}-i \Delta$, $J=\bar{J}-i \Gamma$ ), we get the exact FREs:

$$
\begin{aligned}
& \dot{r}=\Delta / \pi+\Gamma r / \pi+2 r v, \\
& \dot{v}=v^{2}+\bar{\eta}+\bar{J} r+I(t)-\pi^{2} r^{2} .
\end{aligned}
$$

These equations simply contain an extra term $+\Gamma r / \pi$ compared to Eq. (12). Figure 9 shows the bistability boundaries obtained from Eq. (E1) for different ratios of $\Gamma$ and $\Delta$, and $I(t)=0$. Note that the region of bistability shifts to lower values of $\bar{\eta} / \Delta$ and to higher values of $\bar{J} / \sqrt{\Delta}$ as the level of heterogeneity in the synaptic coupling $\Gamma$ is increased.

\section{Firing-rate equations for a pair of excitatory-inhibitory populations}

The microscopic state of each population of QIF neurons is characterized by the membrane potentials $\left\{V_{j}^{(e, i)}\right\}_{j=1, \ldots, N}$, which obey the following ordinary differential equations:

$\dot{V}_{j}^{(e, i)}=\left(V_{j}^{(e, i)}\right)^{2}+I_{j}^{(e, i)}$, if $V_{j}^{(e, i)} \geq V_{p}$, then $V_{j}^{(e, i)} \leftarrow V_{r}$.

Here, $V_{j}^{(e, i)}$ represents the membrane potential of neuron $j$ in either the excitatory $(e)$ or the inhibitory $(i)$ population. The external currents for the excitatory and inhibitory populations are, respectively,

$$
\begin{gathered}
I_{j}^{(e)}=\eta_{j}^{(e)}+J_{e e} s^{(e)}(t)-J_{i e} s^{(i)}(t)+I^{(e)}(t), \\
I_{j}^{(i)}=\eta_{j}^{(i)}+J_{e i} s^{(e)}(t)-J_{i i} s^{(i)}(t)+I^{(i)}(t),
\end{gathered}
$$

where the synaptic weights are $J_{e e}, J_{i i}, J_{i e}, J_{e i}$. Finally, the mean synaptic activation for each population is 


$$
s^{(e, i)}(t)=\frac{1}{N} \sum_{j=1}^{N} \sum_{k \backslash\left(t_{j}^{k}\right)(e, i)<t} \int_{-\infty}^{t} d t^{\prime} a_{\tau}\left(t-t^{\prime}\right) \delta\left(t^{\prime}-\left(t_{j}^{k}\right)^{(e, i)}\right) .
$$

Here, $\left(t_{j}^{k}\right)^{(e, i)}$ is the time of the $k$ th spike of the $j$ th neuron in either the excitatory $(e)$ or the inhibitory $(i)$ population. Additionally, $\delta(t)$ is the Dirac delta function, and $a_{\tau}(t)$ is the normalized synaptic activation caused by a single presynaptic spike with time scale $\tau$, e.g., $a_{\tau}(t)=e^{-t / \tau} / \tau$.

It is straightforward to apply the LA and the method described in the main text to obtain the firing-rate equations corresponding to the two-population model. Considering the limit of infinitely fast synapses, $\tau \rightarrow 0$, we get $s^{(e, i)}(t)=r^{(e, i)}(t)$. Finally, assuming the Lorentzian distributions of currents for both populations,

$$
g_{e, i}(\eta)=\frac{1}{\pi} \frac{\Delta_{e, i}}{\left(\eta-\bar{\eta}_{e, i}\right)^{2}+\Delta_{e, i}^{2}},
$$

we obtain the firing-rate equations:

$$
\begin{aligned}
& \dot{r}^{(e)}=\Delta_{e} / \pi+2 r^{(e)} v^{(e)}, \\
& \dot{v}^{(e)}=\left(v^{(e)}\right)^{2}+\bar{\eta}_{e}+J_{e e} r^{(e)}-J_{i e} r^{(i)}+I^{(e)}(t)-\left(\pi r^{(e)}\right)^{2}, \\
& \dot{r}^{(i)}=\Delta_{i} / \pi+2 r^{(i)} v^{(i)}, \\
& \dot{v}^{(i)}=\left(v^{(i)}\right)^{2}+\bar{\eta}_{i}+J_{e i} r^{(e)}-J_{i i} r^{(i)}+I^{(i)}(t)-\left(\pi r^{(i)}\right)^{2} .
\end{aligned}
$$

[1] B. B. Averbeck, P. E. Latham, and A. Pouget, Neural correlations, population coding and computation, Nat. Rev. Neurosci. 7, 358 (2006).

[2] A. L. Hodgkin and A. F. Huxley, A quantitative description of membrane current and its application to conduction and excitation in nerve, J. Physiol. 117, 500 (1952).

[3] R. Brette, M. Rudolph, T. Carnevale, M. Hines, D. Beeman, J. M. Bower, M. Diesmann, A. Morrison, P. H. Goodman, F. C. Harris, Jr. et al., Simulation of networks of spiking neurons: A review of tools and strategies, J. Comput. Neurosci. 23, 349 (2007).

[4] E. M. Izhikevich and G. M. Edelman, Large-scale model of mammalian thalamocortical systems, Proc. Natl. Acad. Sci. U.S.A. 105, 3593 (2008).

[5] E. M. Izhikevich, Dynamical systems in neuroscience (MIT Press, Cambridge, MA, 2007).

[6] H. R. Wilson and J. D. Cowan, Excitatory and inhibitory interactions in localized populations of model neurons, Biophys. J. 12, 1 (1972).

[7] S.-i. Amari, A method of statistical neurodynamics, Kybernetik 14, 201 (1974).

[8] W. J. Freeman, Mass action in the nervous system (Academic Press, New York, 1975).

[9] W. Gerstner, Time structure of the activity in neural network models, Phys. Rev. E 51, 738 (1995).
[10] S. Fusi and M. Mattia, Collective behavior of networks with linear (VLSI) integrate-and-fire neurons, Neural Comput. 11, 633 (1998).

[11] D. J. Amit and N. Brunel, Model of global spontaneous activity and local structured activity during delay periods in the cerebral cortex, Cereb. Cortex 7, 237 (1997).

[12] N. Brunel, Dynamics of sparsely connected networks of excitatory and inhibitory spiking neurons, J. Comput. Neurosci. 8, 183 (2000).

[13] P. Dayan and L. F. Abbott, Theoretical neuroscience (MIT Press, Cambridge, MA, 2001).

[14] W. Gerstner and W. M. Kistler, Spiking neuron models: Single neurons, populations, plasticity (Cambridge University Press, Cambridge, England, 2002).

[15] O. Shriki, D. Hansel, and H. Sompolinsky, Rate models for conductance-based cortical neuronal networks, Neural Comput. 15, 1809 (2003).

[16] S. Coombes, Waves, bumps, and patterns in neural field theories, Biol. Cybern. 93, 91 (2005).

[17] G. Deco, V. K. Jirsa, P. A. Robinson, M. Breakspear, and K. Friston, The dynamic brain: From spiking neurons to neural masses and cortical fields, PLoS Comput. Biol. 4, e1000092 (2008).

[18] G. B. Ermentrout and D. H. Terman, Mathematical foundations of neuroscience (Springer, New York, 2010), Vol. 64.

[19] S. Ostojic and N. Brunel, From spiking neuron models to linear-nonlinear models, PLoS Comput. Biol. 7, e1001056 (2011).

[20] E. S. Schaffer, S. Ostojic, and L. F. Abbott, A complexvalued firing-rate model that approximates the dynamics of spiking networks, PLoS Comput. Biol. 9, e1003301 (2013).

[21] J. J. Hopfield, Neurons with graded response have collective computational properties like those of two-state neurons, Proc. Natl. Acad. Sci. U.S.A. 81, 3088 (1984).

[22] G. Mongillo, O. Barak, and M. Tsodyks, Synaptic theory of working memory, Science 319, 1543 (2008).

[23] R. Ben-Yishai, R. Lev Bar-Or, and H. Sompolinsky, Theory of orientation tuning in visual cortex, Proc. Natl. Acad. Sci. U.S.A. 92, 3844 (1995).

[24] D. Hansel and H. Sompolinsky, in Methods in neuronal modelling: From ions to networks, edited by C. Koch and I. Segev (MIT Press, Cambridge, MA, 1998), pp. 499-567.

[25] R. Moreno-Bote, J. Rinzel, and N. Rubin, Noise-induced alternations in an attractor network model of perceptual bistability, J. Neurophysiol. 98, 1125 (2007).

[26] K. Zhang, Representation of spatial orientation by the intrinsic dynamics of the head-direction cell ensemble: A theory, J. Neurosci. 16, 2112 (1996).

[27] K.-F. Wong and X.-J. Wang, A recurrent network mechanism of time integration in perceptual decisions, J. Neurosci. 26, 1314 (2006).

[28] M. Abeles, Corticonics: Neural Circuits of the Cerebral Cortex (Cambridge University Press, Cambridge, England, 1991).

[29] F. Varela, J.-P. Lachaux, E. Rodriguez, and J. Martinerie, The brainweb: Phase synchronization and large-scale integration, Nat. Rev. Neurosci. 2, 229 (2001).

[30] A. K. Engel and W. Singer, Temporal binding and the neural correlates of sensory awareness, Trends Cogn. Sci. 5, 16 (2001). 
[31] L. M. Ward, Synchronous neural oscillations and cognitive orocesses, Trends Cogn. Sci. 7, 553 (2003).

[32] P. Fries, A mechanism for cognitive dynamics: Neuronal communication through neuronal coherence, Trends Cogn. Sci. 9, 474 (2005).

[33] G. Buzsaki, Rhythms of the Brain (Oxford University Press, New York, 2006).

[34] J. Fell and N. Axmacher, The role of phase synchronization in memory processes, Nat. Rev. Neurosci. 12, 105 (2011).

[35] E. Ott and T. M. Antonsen, Low dimensional behavior of large systems of globally coupled oscillators, Chaos 18, 037113 (2008).

[36] A. L. Hodgkin, The local electric changes associated with repetitive action in a non-medullated axon, J. Physiol. 107, 165 (1948).

[37] J. Rinzel and B. Ermentrout, in Methods in Neuronal Modelling: From Ions to Networks, edited by C. Koch and I. Segev (MIT Press, Cambridge, MA, 1998), pp. 251-291.

[38] B. Ermentrout and N. Kopell, Parabolic bursting in an excitable system coupled with a slow oscillation, SIAM J. Appl. Math. 46, 233 (1986).

[39] P. E. Latham, B. J. Richmond, P. G. Nelson, and S. Nirenberg, Intrinsic dynamics in neuronal networks. I. Theory, J. Neurophysiol. 83, 808 (2000).

[40] M. A. Buice and C. C. Chow, Dynamic finite size effects in spiking neural networks, PLoS Comput. Biol. 9, e1002872 (2013).

[41] We make an analytic continuation of $w(\eta, t)$ from real $\eta$ into complex-valued $\eta=\eta_{r}+i \eta_{i}$. This is possible into the lower half-plane $\eta_{i}<0$, since this guarantees that the half-width $x(\eta, t)$ remains positive zero: $\partial_{t} x(\eta, t)=-\eta_{i}>0$ at $x=0$. Therefore, we close the integrals in Eqs. (7) and (9) with an arc $|\eta| e^{i \vartheta}$, with $|\eta| \rightarrow \infty$ and $\vartheta \in(-\pi, 0)$. This contour encloses one pole of the Lorentzian distribution [Eq. (11)], which, written in partial fractions, reads as follows: $g(\eta)=(2 \pi i)^{-1}\left[(\eta-\bar{\eta}-i \Delta)^{-1}-(\eta-\bar{\eta}+i \Delta)^{-1}\right]$.

[42] The number of effective parameters can be reduced by nondimensionalizing the system as $\tilde{\eta}=\bar{\eta} / \Delta, \tilde{J}=J / \sqrt{\Delta}$, $(\tilde{r}, \tilde{v})=(r, v) / \sqrt{\Delta}, \tilde{t}=\sqrt{\Delta} t$, and $\tilde{I}(\tilde{t})=I(t / \sqrt{\Delta}) / \Delta$.

[43] Y. Kuramoto, Chemical Oscillations, Waves, and Turbulence (Springer-Verlag, Berlin, 1984).

[44] A. S. Pikovsky, M. G. Rosenblum, and J. Kurths, Synchronization, A Universal Concept in Nonlinear Sciences (Cambridge University Press, Cambridge, England, 2001).

[45] E. Ott and T. M. Antonsen, Long time evolution of phase oscillator systems, Chaos 19, 023117 (2009); E. Ott, B. R. Hunt, and T.M. Antonsen, Comment on "long time evolution of phase oscillators systems", Chaos 21, 025112 (2011).

[46] D. Pazó and E. Montbrió, Low-Dimensional Dynamics of Populations of Pulse-Coupled Oscillators, Phys. Rev. X 4, 011009 (2014).

[47] A. Pikovsky and M. Rosenblum, Dynamics of heterogeneous oscillator ensembles in terms of collective variables, Physica (Amsterdam) 240D, 872 (2011).

[48] T. B. Luke, E. Barreto, and P. So, Complete classification of the macroscopic behavior of a heterogeneous network of theta neurons, Neural Comput. 25, 3207 (2013).
[49] P. So, T. B. Luke, and E. Barreto, Networks of theta neurons with time-varying excitability: Macroscopic chaos, multistability, and final-state uncertainty, Physica (Amsterdam) 267D, 16 (2014).

[50] C. R. Laing, Derivation of a neural field model from a network of theta neurons, Phys. Rev. E 90, 010901 (2014).

[51] E. Montbrió and D. Pazó, Shear Diversity Prevents Collective Synchronization, Phys. Rev. Lett. 106, 254101 (2011).

[52] D. Pazó and E. Montbrió, The Kuramoto model with distributed shear, Europhys. Lett. 95, 60007 (2011).

[53] E. Montbrió and D. Pazó, Collective synchronization in the presence of reactive coupling and shear diversity, Phys. Rev. E 84, 046206 (2011).

[54] D. Iatsenko, S. Petkoski, P. V. E. McClintock, and A. Stefanovska, Stationary and Traveling Wave States of the Kuramoto Model with an Arbitrary Distribution of Frequencies and Coupling Strengths, Phys. Rev. Lett. 110, 064101 (2013).

[55] D. Iatsenko, P. V. E. McClintock, and A. Stefanovska, Oscillator glass in the generalized Kuramoto model: Synchronous disorder and two-step relaxation, Nat. Commun. 5, 4188 (2014).

[56] However, note that the phase diagrams in Luke et al. [48] do not fully agree with ours and show additional instabilities. These bifurcations are associated with the finite width of the pulses, as it occurs in the Winfree model [46].

[57] H. Daido, Onset of cooperative entrainment in limit-cycle oscillators with uniform all-to-all interactions: Bifurcation of the order function, Physica (Amsterdam) 91D, 24 (1996).

[58] Equation (10) then becomes a reversible system [59] of ordinary differential equations with the invariance under time reversal and $w \rightarrow w^{*}$, e.g., Eq. (12) for $\Delta=0$ is reversible, with invariance under $t \rightarrow-t, v \rightarrow-v$. It is advisable to approximate Eq. (10) by a set of ordinary differential equations (one for each $\eta$ value) with additional small terms breaking time reversibility. This is achieved by including a small term in each ordinary differential equation, like $\Delta$ in Eq. (12a), so that $g(\eta)$ is actually approximated by a sum of very sharp Lorentzian functions.

[59] J. A. G. Roberts and G. R. W. Quispel, Chaos and time-reversal symmetry. Order and chaos in reversible dynamical systems, Phys. Rep. 216, 63 (1992).

[60] S. Watanabe and S. H. Strogatz, Constant of motion for superconducting Josephson arrays, Physica (Amsterdam) 74D, 197 (1994).

[61] A. Pikovsky and M. Rosenblum, Partially integrable dynamics of hierarchical populations of coupled oscillators, Phys. Rev. Lett. 101, 264103 (2008).

[62] A subtle question is that, as it occurs in the Kuramoto model, there exists a continuous spectrum of eigenvalues at $\lambda=-2 w_{0}(\eta) i$ and the complex conjugate. Indeed, for $\eta$ values such that neurons are actively firing $\left[y_{0}(\eta)=0\right]$, the eigenvalues lie exactly on the imaginary axis. Apparently, this seems to forbid the exponential stability obtained from Eq. (12) for the Lorentzian distribution. The same mathematical conundrum was encountered and solved in the 
Kuramoto model [63-65], which suggests that similar mechanisms are operating here.

[63] S. H. Strogatz and R. E. Mirollo, Stability of incoherence in a population of coupled oscillators, J. Stat. Phys. 63, 613 (1991).

[64] S. H. Strogatz, R. E. Mirollo, and P. C. Matthews, Coupled Nonlinear Oscillators below the Synchronization
Threshold: Relaxation by Generalized Landau Damping, Phys. Rev. Lett. 68, 2730 (1992).

[65] H. Chiba and I. Nishikawa, Center manifold reduction for large populations of globally coupled phase oscillators, Chaos 21, 043103 (2011).

[66] Note that considering stronger forms of heterogeneity for the synaptic weights may generally not lead to Eq. (19). 